\title{
Effect of the boundary shape in the effective theory of fractional quantum Hall edges
}

\author{
D. C. Cabra ${ }^{1, *}$ and N. E. Grandi ${ }^{2,}$ \\ ${ }^{1}$ Laboratoire de Physique Théorique, Université Louis Pasteur, 3 rue de l'Université, F-67084 Strasbourg Cedex, France; \\ Departamento de Física, Universidad Nacional de la Plata, Casilla de Correos 67, (1900) La Plata, Argentina; \\ and Facultad de Ingeniería, Universidad Nacional de Lomas de Zamora, Camino de Cintura y Juan XXIII, (1832) \\ Lomas de Zamora, Argentina \\ ${ }^{2}$ Departamento de Física, Universidad Nacional de la Plata, Casilla de Correos 67, (1900) La Plata, Argentina
}

(Received 8 June 2007; revised manuscript received 7 November 2007; published 6 March 2008)

Starting from a microscopic description of a system of strongly interacting electrons in a strong magnetic field in a finite geometry, we construct the boundary low energy effective theory for a fractional quantum Hall droplet taking into account the effects of a smooth edge. The effective theory obtained is the standard chiral boson theory (chiral Luttinger theory) with an additional self-interacting term which is induced by the boundary. As an example of the consequences of this model, we show that such modification leads to a nonuniversal reduction in the tunneling exponent which is independent of the filling fraction. This is in qualitative agreement with experiments, which systematically found exponents smaller than those predicted by the ordinary chiral Luttinger liquid theory.

DOI: 10.1103/PhysRevB.77.115107

PACS number(s): 71.10.-w, 73.43.Jn, 73.43.Lp

\section{INTRODUCTION}

Quantum Hall effect (QHE) has been the subject of intense research since 1980 when the first plateaux in the Hall conductivity were observed for integer filling fractions. ${ }^{1}$ The interest on these systems was then reinforced after the fractional QHE (FQHE) was identified as a novel state of matter. ${ }^{2}$ Since then, different approaches have been developed and applied to the study of these systems, such as the successful variational ground-state wave function proposed by Laughlin. ${ }^{3}$ This description led to the concept of fractionally charged quasiparticles as a consequence of the strong correlations in the system, which were in turn experimentally observed. ${ }^{4}$ The composite fermion approach, ${ }^{5}$ which provides a description of the FQHE as a QHE where a quasiparticle is built up as a bound state of an electron and a given number of magnetic flux quanta, also provides a consistent description. An alternative description of these systems, based on the Chern-Simons topological action, also reproduces many of the experimentally observed features. ${ }^{6}$

In spite of the enormous success of all these descriptions, a theory for the QHE derived systematically from a microscopic description is still lacking. It is the purpose of the present paper to give one step in this direction and provide an effective description of the FQHE which is derived from a first principles microscopic action describing interacting electrons in a strong external magnetic field in a controlled way. In doing this, we are able to complement previous descriptions and provide a way to take into account the effects of a smooth boundary. These effects have been already considered in Refs. 7 and 10-15; the main difference with our approach is that our starting point is the microscopic model and hence it does not rely on a phenomenological description.

The study of tunneling of electrons into incompressible and compressible quantum Hall states has been the subject of intense research from both the theoretical and experimental sides (see Ref. 7 and references therein). It is found that the tunneling conductivity is non-Ohmic, $I \propto V^{\alpha}$, with $\alpha$ being a function of the filling fraction $\nu$. This behavior can be understood within the chiral Luttinger liquid description of the edge physics advanced by Wen. ${ }^{9}$ Although tunneling experiments in FQHE systems have shown certain degree of agreement with the theoretical predictions obtained from the chiral Luttinger liquid picture, ${ }^{9}$ there remain certain discrepancies which have been addressed by different authors. ${ }^{7,10-15}$ In particular, a reduction of the order of $10 \%$ from the theoretical prediction for the tunnelling exponent has been observed experimentally, as well as the absence of the theoretically predicted plateaux structure of this exponent. ${ }^{10,13,15}$ These issues have been the subject of intense debate. ${ }^{7}$

Here we present an alternative derivation of the boundary effective action, by including higher order terms in the low energy expansion which are induced by the smoothness of the edge. This paper can be considered as a formal derivation of the model first presented in Ref. 8. In our calculations, the presence of a smooth boundary shows to be crucial, e.g., in the computation of tunneling exponents. The effects of the edge shape and the confining potential on these observables have been recently addressed using numerical exact diagonalization, ${ }^{12}$ where it was found that the result could deviate from the chiral Luttinger liquid universal predictions. Our results are in agreement with these findings.

In the simplest case of an almost sharp edge, the effective action we obtain corresponds to a self-interacting chiral boson. Using this improved effective action we compute the tunneling exponent which, to one loop order, receives a small nonuniversal negative correction depending on the electron density and the short distance cutoff (the "effective size" of the particles).

The paper is organized as follows: In Sec. II, we present the model which corresponds to a microscopic description of a fully polarized self-interacting electron gas in a transverse magnetic field. The action is invariant under relabeling of particles, a symmetry that plays an important role in the continuum limit (corresponding to the low energy description) as we show in Sec. IV. In Sec. III we rewrite the action by 
making a boost transformation to eliminate the external electric field and we discard terms quadratic in the particle velocities. This last approximation is justified due to the presence of a strong external magnetic field. We show that as a result of the interactions, the system is projected into the fundamental state and that this projection corresponds to the imposition of a constraint (Sec. III B) which defines the effective degrees of freedom as the fluctuations along the flat directions of the potential. In Sec. IV we construct the low energy effective action by going to the continuum limit and show that the resulting action is topological. We show that the original symmetry under relabeling of particles corresponds to the invariance under area preserving diffeomorphisms of the effective action. A condition which arises from the solution of the constraint is that our analysis is applicable to incompressible states (Sec. IV D). Although the constituent particles are fermions, the collective degrees of freedom, which correspond to fluctuations along the flat directions of the interaction potential, are described by bosonic fields.

In Sec. $\mathrm{V}$ we first solve the constraint by performing a gradient expansion on the coordinate fields and then, by boosting back to the original (laboratory) coordinate system, we arrive at one of our main results, which is the effective action (5.5). In Sec. VI we look in more detail into the effective action close to a smooth boundary, which requires to recover the discrete structure along the direction perpendicular to the boundary, as expected. This leads generically to a boundary effective theory of $\mathcal{N}$ coupled chiral bosons, with $\mathcal{N}=W / a, a$ being the mean interparticle distance and $W$ the effective width of the edge (6.1). By integrating out the internal degrees of freedom, we finally obtain the effective action for the boundary modes, which corresponds to that of a chiral self-interacting bosonic field. From this action, we compute the correction to the tunneling exponent, the result being in qualitative agreement with experiments. The result obtained for the exponent is nonuniversal, which is a direct consequence of the inclusion of a smooth edge. ${ }^{16}$ A higher loop computation together with a renormalization group analysis should be done, but this is out of the scope of the present paper. The main aim here is to obtain an effective field theory, starting from a microscopic description of the real problem and which includes the effects of smooth boundary, a feature present in almost all experiments. The observed edge reconstruction effect ${ }^{14}$ could be in principle recovered within our approach by including the effects of the fluctuation fields perpendicular to the flat directions of the interaction potential [field $\Omega$ in Eq. (4.12)]. This is the subject of future investigations. We conclude in Sec. VII with a discussion of the results and open perspectives.

\section{POLARIZED ELECTRON GAS IN A TRANSVERSE MAGNETIC FIELD}

\section{A. Action}

The most general action for a system of $N$ interacting electrons is given by

$$
S=\int d t\left(\sum_{p}^{N} \frac{m}{2} \dot{\mathrm{x}}_{p}^{i 2}-V\left[\mathrm{x}_{q}^{i}, \dot{\mathrm{x}}_{q}^{i}\right]\right), \quad i=1,2, \quad p, q=1, \ldots, N,
$$

where the degrees of freedom of the system are represented by the $2 N$ functions $x_{p}^{i}(t)$, i.e., the positions of the electrons in a Cartesian coordinate system. These functions will be our dynamical variables. For our purposes it is enough to consider spinless fermions in two space dimensions since, as explained later, typical experiments are performed in the presence of strong magnetic fields.

Note that this action is completely generic and makes no simplification. The electrons interact with the background and with each other by means of a general potential $V\left[\mathrm{x}_{p}, \dot{\mathrm{x}}_{p}\right]$. This potential may depend simultaneously on the positions and velocities of all particles (i.e., all $p=1, \ldots, N$ ), and to keep this in mind we write its argument in square brackets.

We split the interaction into an external part and an electron-electron interaction part $V\left[\mathrm{x}_{p}, \dot{\mathrm{x}}_{p}\right]=V^{\text {ext }}\left[\mathrm{x}_{p}, \dot{\mathrm{x}}_{p}\right]$ $+V^{i n t}\left[\mathrm{x}_{p}, \dot{\mathrm{x}}_{p}\right]$. Since electrons interact independently with the external field, the external part can be written as a sum of a single term per electron $V^{\text {ext }}\left[\mathrm{x}_{p}, \dot{\mathrm{x}}_{p}\right]=\sum_{p}^{N} V^{\text {ext }}\left(\mathrm{x}_{p}, \dot{\mathrm{x}}_{p}\right)$ and we then have

$$
S=\int d t\left(\sum_{p}^{N}\left(\frac{m}{2} \dot{\mathrm{x}}_{p}^{i 2}-V^{e x t}\left(\mathrm{x}_{p}^{i}, \dot{\mathrm{x}}_{p}^{i}\right)\right)-V^{i n t}\left[\mathrm{x}_{q}^{i}, \dot{\mathrm{x}}_{q}^{i}\right]\right),
$$

where the first term corresponds to the kinetic contribution.

Since the system is nonrelativistic, the external potential $V^{e x t}\left(\mathrm{x}_{p}^{i}, \dot{\mathrm{x}}_{p}^{i}\right)$ can be expanded in powers of velocities as

$$
V^{e x t}\left(\mathrm{x}_{p}^{i}, \dot{\mathrm{x}}_{p}^{i}\right)=u\left(\mathrm{x}_{p}^{i}\right)+e A_{j}\left(\mathrm{x}_{p}^{i}\right) \dot{\mathrm{x}}_{p}^{j}+\frac{1}{2} \delta m\left(\mathrm{x}_{p}^{j}\right) \mathrm{x}_{p}^{i 2}+\mathcal{O}\left(\dot{\mathrm{x}}_{p}^{i 3}\right)
$$

the first two terms representing the electric and magnetic coupling to the external field. The quadratic order in velocities represents a position dependent correction to the mass that can be reabsorbed in the kinetic term, while higher orders have been discarded.

On the other hand, the expansion of the interparticle interaction $V^{i n t}\left[\mathrm{x}_{p}^{i}, \dot{\mathrm{x}}_{p}^{i}\right]$ reads

$$
\begin{aligned}
V^{i n t}\left[\mathrm{x}_{p}^{i}, \dot{\mathrm{x}}_{p}^{i}\right]= & V^{i n t}\left[\mathrm{x}_{p}^{i}, 0\right]+\sum_{q}^{N} \frac{\partial V^{i n t}}{\partial \dot{\mathrm{x}}_{q}^{j}}\left[\mathrm{x}_{p}^{i}, 0\right] \dot{\mathrm{x}}_{q}^{j} \\
& +\sum_{q}^{N} \sum_{r}^{N} \frac{\partial^{2} V^{i n t}}{\partial \dot{\mathrm{x}}_{q}^{j} \partial \mathrm{x}_{r}^{k}}\left[\mathrm{x}_{p}^{i}, 0\right] \dot{\mathrm{x}}_{q}^{j} \dot{\mathrm{x}}_{r}^{k}+\mathcal{O}\left(\dot{\mathrm{x}}^{i 3}\right) .
\end{aligned}
$$

The resulting terms can be grouped according to the power of velocities involved, resulting in a masslike (particle mixing) term, a magnetic term linear in velocities, and an electric potential term 


$$
\begin{aligned}
& S=\int d t\left(\frac{1}{2} \sum_{p, q}^{N}\left(\left(m+\delta m\left(\mathrm{x}_{p}^{j}\right)\right) \delta_{p q}-\frac{\partial^{2} V^{i n t}}{\partial \dot{\mathrm{x}}_{p}^{j} \partial \dot{\mathrm{x}}_{q}^{k}}\left[\mathrm{x}_{r}^{i}, 0\right]\right) \dot{\mathrm{x}}_{p}^{j} \dot{\mathrm{x}}_{q}^{k}\right. \\
& \left.-\sum_{p}^{N}\left(e A_{j}\left(\mathrm{x}_{p}^{i}\right)+\frac{\partial V^{i n t}}{\partial \dot{\mathrm{x}}_{p}^{j}}\left[\mathrm{x}_{q}^{i}, 0\right]\right) \dot{\mathrm{x}}_{p}^{j}-\sum_{p}^{N} u\left(\mathrm{x}_{p}^{i}\right)-V^{i n t}\left[\mathrm{x}_{p}^{i}, 0\right]\right) .
\end{aligned}
$$

This is the action of our system. Up to this point no approximation has been made (other than the nonrelativistic limit) and the action has been kept completely general.

\section{B. Important symmetry}

Since the particles are identical, the action is naturally invariant under arbitrary permutations of the particle indices. We will call this property invariance under relabeling of the particles. This can be formally formulated as follows: the redefinition

$$
p \rightarrow p^{\prime}=p^{\prime}(p)
$$

acts in the dynamical variables as

$$
\mathrm{x}_{p}^{i}=\mathrm{x}_{p\left(p^{\prime}\right)}^{i} \equiv \mathrm{x}_{p^{\prime}}^{i \prime}
$$

leaving the action unchanged.

\section{INTERACTIONS AND THE RESULTING PROJECTION INTO THE FUNDAMENTAL STATE}

The general expansion for the external potential (2.3) resulted in an electromagnetic interaction in three space-time dimensions. We define the corresponding external magnetic field as $\vec{B}=B \check{z}$, with $B=\partial_{i} A_{j} \epsilon^{i j}$ and we assume it is constant and homogeneous in all space to conform with the typical experimental-setup. Moreover, we take it to be very strong so as to completely polarize the particle spin in the $\check{z}$ direction, justifying in this way our choice of spinless fermions in two dimensions to represent the electrons. The electric field on the other hand is defined as $E_{i}=-\partial_{i} u$. It is assumed to be zero in the interior of the region of space occupied by the sample, and smoothly growing at the edge of that region.

\section{A. Convenient coordinate system}

In three dimensions, the $3 \times 3$ antisymmetric matrix representing the electromagnetic tensor $F_{\mu \nu}$ has at least one zero eingenvalue. Then it can be taken into its block diagonal form by a curvilinear change of coordinates $\mathrm{x}^{i} \rightarrow \widetilde{\mathrm{x}}^{i}(\mathrm{x}, t)$. In consequence, in the new coordinate system there is no electric field and we can choose a gauge in which $u(\widetilde{\mathrm{x}})=0$. It can be shown by a straightforward calculation that such change of variables satisfies

$$
\partial_{t} x^{i}=-\frac{1}{B} \epsilon^{i j} \partial_{j} u \equiv v_{i}, \quad \partial_{t} t=1 .
$$

We can solve for the magnetic field in the boosted coordinate system $\widetilde{B}$ as

$$
\widetilde{B} \simeq B\left(1-\frac{\left(\partial_{i} u\right)^{2}}{2 B^{2}}\right) .
$$

The action in the new coordinate system reads

$$
\begin{aligned}
S= & \int d t\left(\frac{1}{2} \sum_{p, q}^{N}\left(\left(m+\delta m\left(\tilde{\mathrm{x}}_{p}^{j}\right)\right) \delta_{p q}-\frac{\partial^{2} V^{i n t}}{\partial \dot{\tilde{\mathrm{x}}}_{p}^{j} \partial \dot{\overline{\mathrm{x}}}_{q}^{k}}\right) \dot{\widetilde{\mathrm{x}}}_{p}^{j} \dot{\overline{\mathrm{x}}}_{q}^{k}\right. \\
& \left.-\sum_{p}^{N}\left(e \widetilde{A}_{j}\left(\widetilde{\mathrm{x}}_{p}^{i}\right)+\frac{\partial V^{i n t}}{\partial \dot{\tilde{\mathrm{x}}}_{p}^{j}}\right) \dot{\tilde{\mathrm{x}}}_{p}^{j}-V^{i n t}\right),
\end{aligned}
$$

where $\widetilde{A}_{j}\left(\widetilde{\mathrm{x}}_{p}^{i}\right)$ is the external vector potential in the new coordinates. Note that in this frame $\tilde{u}\left(\widetilde{\mathrm{x}}_{p}^{i}\right)=0$ and the external magnetic field $\widetilde{B}\left(\widetilde{\mathrm{x}}_{p}^{i}\right)$ is nonhomogeneous. In other words, the effects of the external electric field in the old coordinate system $\mathrm{x}$ are now encoded in $\widetilde{B}\left(\widetilde{\mathrm{x}}_{p}^{i}\right)$ in the new system $\widetilde{\mathrm{x}}_{p}^{i}$. This is very important since, as we will see, it turns out to lead to localization of the excitations.

On the other hand, it is evident that the relabeling symmetry persists in the new variables, taking the same form as before, i.e.,

$$
\widetilde{\mathrm{x}}_{p}^{i}=\widetilde{\mathrm{x}}^{\prime i}{ }^{\prime} \cdot
$$

\section{B. Projection constraint and a new form of the action}

Due to the presence of a strong external magnetic field, we can discard the kinetic terms quadratic in velocities, as well as the magnetic interparticle interaction $\left(\partial V^{i n t} / \partial \dot{\mathrm{x}}_{p}^{j}\right) \dot{\mathrm{x}}_{p}^{j}$. In a perturbative quantization approach, this approximation corresponds to the projection into the lowest Landau level (LLL). The scrupulous reader can keep all the terms, then go through a quantization procedure, and project the result into the LLL.

The resulting effective Lagrangian is then linear in velocities and the resulting action for the degrees of freedom in the ground state is

$$
S=\int d t\left(\sum_{p}^{N} e \widetilde{A}_{j}\left(\widetilde{\mathrm{x}}_{p}^{i}\right) \dot{\widetilde{\mathrm{x}}}_{p}^{j}-V^{i n t}\left[\widetilde{\mathrm{x}}_{p}^{i}\right]\right),
$$

where we called $V^{i n t}\left[\widetilde{\mathrm{x}}_{p}^{i}\right]=V^{i n t}\left[\mathrm{x}_{p}^{i}, 0\right]$. To be consistent, we should supplement this action with the condition enforcing the low energy projection, namely, that the system remains in its ground state $\mathcal{H}=E_{\text {min }}$. Within our approximation, this can be written as

$$
V^{i n t}\left[\widetilde{\mathrm{x}}_{p}^{i}\right]=0,
$$

where we have eliminated any nonvanishing contribution to the ground-state energy by a shift in the potential. The consequences of relaxing this assumption will be discussed later. This constraint can be enforced at the action level by introducing a Lagrange multiplier $\lambda(t)$ to obtain

$$
S=\int d t\left(\sum_{p}^{N} e \widetilde{A}_{j}\left(\widetilde{\mathrm{x}}_{p}^{i}\right) \dot{\widetilde{\mathrm{x}}}_{p}^{j}-\lambda V^{i n t}\left[\widetilde{\mathrm{x}}_{p}^{i}\right]\right),
$$

the equations of motion being now obtained by varying the action with respect to $\widetilde{\mathrm{x}}_{p}^{i}$ and the Lagrange multiplier $\lambda(t)$. 
This new form of the action is still invariant under the relabeling symmetry.

\section{CONTINUUM LIMIT AND THE RESULTING TOPOLOGICAL FIELD THEORY}

We assume that all the important physical scales involved in our problem are large compared to the interparticle distance. These scales can be characterized in terms of the relevant length of the external field $\widetilde{B} / \partial_{j} \widetilde{B}$. Since the derivatives involved in this definition can differ significatively when taken in different directions, so does the accuracy of this assumption. In the present section we assume that it is valid in any direction, an assumption which is well justified in the bulk. We will come back to this point in the case in which this assumption ceases to be valid, i.e., when analyzing boundary effects in Sec. VI.

\section{A. Continuum space}

We take the continuum limit in the above system as follows: first we define our continuum space and functions living on it by the following steps.

(1) We introduce an auxiliary two-dimensional space parametrized by variables $\tilde{y}^{i}$.

(2) We set a (not necessarily regular) lattice $\tilde{y}_{p}^{i}$ in that space by the condition $\tilde{y}_{p}^{i}=\widetilde{x}_{p}^{i}(0)$, which will be useful later.

(3) We use the discrete quantities $\tilde{\mathrm{x}}_{p}^{i}(t)$ of our problem to define lattice functions $\widetilde{\mathrm{x}}^{i}\left(\tilde{y}_{p}^{j}, t\right)$ on the auxiliary $\tilde{y}_{p}$ space by means of

$$
\widetilde{\mathrm{x}}^{i}\left(\tilde{y}_{p}^{j}, t\right)=\tilde{\mathrm{x}}_{p}^{i}(t)
$$

In other words, we label each particle $p$ with their initial position $\tilde{y}_{p}^{i}$.

(4) We reinterpret these lattice quantities as the values at the lattice points of some continuum fields $\widetilde{\mathrm{x}}^{i}\left(\tilde{y}^{j}, t\right)$

$$
\widetilde{\mathrm{x}}^{i}\left(\tilde{y}_{p}^{j}, t\right)=\left.\tilde{\mathrm{x}}^{i}\left(\tilde{y}^{j}, t\right)\right|_{y^{i}=y_{p}^{i}} .
$$

These continuum fields can be understood as an interpolation of the discrete quantities representing the particle positions at a given time in the intersticial region of $\tilde{y}$ space. There are many interpolation methods that could be used for that; ${ }^{17}$ however, we do not need an explicit prescription for it. Note that this reinterpretation is consistent with the initial condition on the continuum variables

$$
\left.\widetilde{\mathrm{x}}^{i}\left(\tilde{y}^{j}, t=0\right)\right|_{\tilde{y}^{j}=\tilde{y}_{p}^{j}}=\mathrm{x}^{i}\left(\tilde{y}_{p}^{j}, 0\right)=\widetilde{\mathrm{x}}_{p}^{i}(0)=\tilde{y}_{p}^{i} .
$$

It should be kept in mind that the continuum variables introduced here are useful only as an approximation of the underlying discrete dynamics. Then it will make sense to identify solutions of the equations of motions that differ in regions smaller than the interparticle distance. In other words, wavelengths shorter than that distance should be interpreted as unphysical, limiting the number of degrees of freedom introduced by the continuum limit.

\section{B. Resulting field theory}

We now reformulate the dynamics in terms of the continuum variables introduced in Eq. (4.1) by starting from the discrete action (3.7). The procedure goes as follows.

(1) We make a simplicial decomposition of the $\tilde{y}^{i}$ plane consistent with the lattice, i.e., having a single point $\widetilde{y}_{p}^{i}$ for each simplex. It is easy to convince oneself that this is always possible and that in two dimensions the simplices are triangles that can be defined as having a lattice point at each vertex. The easiest choice is a simplicial decomposition topologically equivalent to the triangular lattice.

(2) We call $\Delta \tilde{A}_{p}$ the (small) area of the $p$ th simplex and multiply and divide by it the $p$ th term in the action (3.7). This results in a Riemann approximation for the integral

$$
\begin{aligned}
& \sum_{p}^{N} e \widetilde{A}_{j}\left(\widetilde{\mathrm{x}}\left(\tilde{y}_{p}^{i}, t\right)\right) \partial_{t} \widetilde{\mathrm{x}}\left(\tilde{y}_{p}^{j}, t\right) \\
& =\sum_{p}^{N} \Delta \tilde{A}_{p} \frac{1}{\Delta \tilde{A}_{p}} e \widetilde{A}_{j}\left(\widetilde{\mathrm{x}}\left(\tilde{y}_{p}^{i}, t\right)\right) \partial_{t} \widetilde{\mathrm{x}}\left(\tilde{y}_{p}^{j}, t\right) \\
& \simeq \int d^{2} \tilde{y} \widetilde{\rho}(\widetilde{y}) e \widetilde{A}_{j}\left(\widetilde{\mathrm{x}}\left(\tilde{y}^{i}, t\right)\right) \partial_{t} \widetilde{\mathrm{x}}\left(\tilde{y}^{j}, t\right) \\
& +\mathcal{O}\left[\sum_{p}\left|\partial_{i}\left(e \tilde{\rho} \widetilde{A}_{j}(\widetilde{\mathrm{x}}) \partial_{t} \widetilde{\mathrm{x}}\right) \Delta y_{p}^{i}\right|_{\left(\widetilde{y}_{p}, t\right)} \Delta \widetilde{A}_{p}\right],
\end{aligned}
$$

where the number density function $\widetilde{\rho}(\widetilde{y})$ stands for the interpolation of $\tilde{\rho}\left(\tilde{y}_{p}\right) \equiv 1 / \Delta \tilde{A}_{p}$. This approximation of the sum remains accurate as long as the jump on the argument when going from one lattice point to another stays small. We have kept terms up to linear order in these jumps and provided an estimation of the error made in that process that will be useful later. Even if we won't write explicitly the error (4.4) on the action in what follows, we will keep in mind that our integrals make sense as long as it is small. Applying the above steps explicitly we obtain the continuum low energy effective theory

$$
S=\int d t\left(\int d^{2} \tilde{y} \tilde{\rho} e \tilde{A}_{j}\left(\widetilde{\mathrm{x}}^{i}\right) \partial_{t} \widetilde{\mathrm{x}}-\lambda V^{i n t}[\tilde{\mathrm{x}}]\right),
$$

in terms of the continuum fields $\tilde{\mathrm{x}}^{i}(\tilde{y}, t)$ and the Lagrange multiplier $\lambda(t)$.

We are interested in solutions $\widetilde{\mathrm{x}}^{i}(\widetilde{y}, t)$ of the field theory (4.5) that are good approximations of the true solutions $\widetilde{\mathrm{x}}_{p}^{i}(t)$ of the discrete microscopic system. In particular, this means that the error estimated in Eq. (4.4) has to be small when evaluated on them. In other words, we should discard those solutions whose wavelengths are small compared to the interparticle distance.

Note that the action contains a local velocity-dependent term, coming from the external magnetic field, plus a nonlocal potential constraint, coming from the interparticle interaction. The topological nature of the resulting field theory is evident in view of the absence of any spatial derivatives. Moreover, to make sense of the constraint, we should make sure that it does not couple long wavelength (physical) modes with short wavelength (unphysical) ones.

In this part of the calculation a new function $\tilde{\rho}(\tilde{y})$ has appeared. We need some suitable ansatz for the form of this function. Since it represents the particle number density in 
the ground state, a judicious ansatz would be to choose it consistently with the forces on these particles in that state. In the $\tilde{y}$ plane, particles at rest experience no forces other than their repulsive interaction, so we can safely choose $\widetilde{\rho}(\widetilde{y})$ as a constant $\tilde{\rho}_{o}$ in this plane.

\section{Symmetry under area preserving diffeomorphisms and gauge invariance}

The relabeling symmetry (2.6), (2.7), and (3.4) is inherited by the continuum limit theory, where it manifests as a symmetry under area preserving diffeomorphisms. To see this, we first note that the area $\Delta \widetilde{A}_{p}$ of the simplex associated to the particle $p$ becomes, after a relabeling,

$$
\Delta \widetilde{A}_{p}=\Delta \tilde{A}_{p\left(p^{\prime}\right)}=\Delta \widetilde{A}_{p^{\prime}}^{\prime} .
$$

The second equality is evident from the fact that $\Delta \widetilde{A}_{p}$ becomes, after relabeling, the area of the simplex associated to the particle $p^{\prime}$.

In the continuum limit, the symmetry can be written as

$$
y \rightarrow \tilde{y}^{\prime}=\tilde{y}^{\prime}(\tilde{y}),
$$

while the fields transform according to

$$
\mathrm{x}^{i^{\prime}}\left(y^{\prime}, t\right)=\mathrm{x}^{i}(y, t) .
$$

The equality (4.6) then implies that

$$
d^{2} \tilde{y}=d^{2} \tilde{y}^{\prime} .
$$

Then the transformation corresponding to the relabeling of particles becomes an area preserving diffeomorphism in the continuum theory

Due to the definition of $\tilde{\rho}$, the combination $\widetilde{\rho} d^{2} \widetilde{y}$ is invariant under any transformation. Then the kinetic term in the action is obviously invariant under area preserving diffeomorphisms. The potential, on the other hand, inherits its invariance from the discrete case.

We conclude that our system has a continuous invariance. In the case in which $\widetilde{B}$ is constant, this corresponds to the gauge invariance of the usual Chern-Simons description. ${ }^{9} \mathrm{On}$ the other hand, in the case in which $\widetilde{B}$ is space dependent, the only transformations leaving the action invariant are those which are time independent. This implies that gauge invariance is broken in our theory, in a similar way as the imposition of a boundary breaks gauge invariance in Ref. 9, enforcing the introduction of the boundary chiral boson. In our formulation this chiral boson theory arises naturally as a consequence of the low energy constraint. This is an insight of one of the main claims of this paper, i.e., that a smooth boundary, represented by an external potential varying in space, can be described by a theory whose qualitative behavior is similar of that of the chiral boson.

\section{Flat directions of the ground state and a simpler form of the constraint}

The Lagrange multiplier in the action imposes the constraint that forces the system to be in the ground state. To obtain the remaining degrees of freedom we would then need to solve this constraint. This seems impossible without knowing explicitly the form of the potential, but we show in this section that this is indeed simplified by the symmetry under area preserving diffeomorphisms of our system. In consequence we can replace the constraint for an explicit one, without giving any details on the potential.

The condition (3.6) fixes all directions except those that leave the potential invariant. In other words, particles can move along the flat directions of the potential, while staying in the ground state. These directions then correspond to the remaining degrees of freedom. To determine them, let us note that any small deformation $\widetilde{\mathrm{x}}^{i}(\widetilde{y}, t)=\widetilde{\mathrm{x}}_{o}^{i}(\widetilde{y}, t)+\delta \widetilde{\mathrm{x}}^{i}(\widetilde{y}, t)$ around a given minimum of the potential $\widetilde{\mathrm{x}}_{o}^{i}(\widetilde{\mathrm{y}}, t)$ can be written in the form

$$
\delta \widetilde{\mathrm{x}}^{i}(\tilde{y}, t)=M_{i j}(\tilde{y}, t)\left(\partial_{j} \Omega(\tilde{y}, t)+\epsilon_{j k} \partial_{k} \Lambda(\tilde{y}, t)\right)
$$

for suitable $(\Omega, \Lambda)$, when $M$ is a given invertible matrix field that we choose for convenience as $M_{i j}=\partial_{i} \tilde{\mathrm{x}}_{o}^{j}(\tilde{y}, t)$. With this choice we can write

$$
\delta \widetilde{\mathrm{x}}^{i}(\widetilde{y}, t)=\partial_{i} \widetilde{\mathrm{x}}_{o}^{j}(\widetilde{y}, t)\left(\partial_{j} \Omega(\tilde{y}, t)+\epsilon_{j k} \partial_{k} \Lambda(\tilde{y}, t)\right)
$$

or in other words

$$
\widetilde{\mathrm{x}}^{i}(\tilde{y}, t)=\widetilde{\mathrm{x}}_{o}^{i}\left(\widetilde{y}_{j}+\partial_{j} \Omega(\tilde{y}, t)+\epsilon_{j k} \partial_{k} \Lambda, t\right),
$$

implying that any small deformation of the configuration $\widetilde{\mathrm{x}}_{o}^{j}(\tilde{y}, t)$ can be obtained by a small diffeomorphism on $\tilde{y}$. The $\Omega$ part represents dilatations of the $\tilde{y}$ plane, while the $\Lambda$ part corresponds to area preserving deformations. Going now to the potential, this implies

$$
\begin{aligned}
V\left[\widetilde{\mathrm{x}}_{o}(\tilde{y}, t)+\delta \widetilde{x}^{j}(\tilde{y}, t)\right] & =V\left[\widetilde{\mathrm{x}}_{o}\left(\tilde{y}_{j}+\partial_{j} \Omega+\epsilon_{j k} \partial_{k} \Lambda, t\right)\right] \\
& =V\left[\widetilde{\mathrm{x}}_{o}\left(\tilde{y}_{j}+\partial_{j} \Omega(\tilde{y}, t), t\right)\right],
\end{aligned}
$$

where in the last equality we use the invariance of the potential under the area preserving diffeomorphism generated by $\Lambda, \delta \widetilde{y}_{i}=\epsilon_{i j} \partial_{j} \Lambda$. We conclude that the condition (3.6) fixes $\Omega$ and leaves $\Lambda$ completely free. The flat directions of the potential are then the variations of $\widetilde{x}_{o}^{i}(\widetilde{y}, t)$ generated by area preserving diffeomorphisms.

In particular, since $\widetilde{x}^{i}(\tilde{y}, 0)=\widetilde{y}^{i}$, we conclude that the time evolution map, going from $\tilde{y}^{i}$ to $\widetilde{\mathrm{x}}^{i}(\tilde{y}, t)$, is area preserving. In other words, the Jacobian of the time evolution is unity, implying that a physical configuration satisfies

$$
\left|\partial_{i} \widetilde{\mathrm{x}}^{j}\right|=1 .
$$

This constraint has a simple and intuitive interpretation: note that the particle density (in $\widetilde{\mathrm{x}}^{i}$ space) at time $t$ is given in terms of the initial density $\widetilde{\rho}_{o}$ by the formula

$$
\widetilde{\rho}(t, \widetilde{\mathrm{x}})=\frac{\widetilde{\rho}_{o}}{\left|\partial_{i} \widetilde{\mathrm{x}}^{j}\right|} \equiv \tilde{\rho}_{o},
$$

i.e., our evolution is that of an incompressible fluid. For this reason, we will concentrate in what follows in incompressible states. To describe compressible states on the other hand, we would have to consider the $\Omega$ degree of freedom, or in other words relaxing the assumption (3.6). Some insights on this will be given later. 
It should be clear now that we can replace the constraint (3.6) by the more explicit one (4.14). In other words, we can replace Eq. (4.5) by the completely local action

$$
S=\int d t \int d^{2} \tilde{y} \widetilde{\rho}_{o}\left(e \widetilde{A}_{j}\left(\widetilde{\mathrm{x}}^{i}\right) \partial_{t} \widetilde{\mathrm{x}}-\lambda\left(\left|\partial_{i} \widetilde{\mathrm{x}}^{j}\right|-1\right)\right),
$$

where now $\lambda(\tilde{y}, t)$ is a local Lagrange multiplier imposing Eq. (4.14).

As a conclusion, in this section we have been able to use the symmetries to describe the low energy dynamics independently of the explicit form of the interparticle potential.

\section{E. Conserved charge and an immediate consequence}

Since the action is invariant under the group of area preserving diffeomorphisms in the $\tilde{y}^{i}$ plane, we can calculate the corresponding Nœther charge. To do that, we write the infinitesimal transformation as

$$
y_{i}^{\prime}=y_{i}+\epsilon_{i j} \partial_{j} \Lambda
$$

and the charge reads

$$
Q=\frac{e}{2} \int d^{2} y \widetilde{B}(\widetilde{\mathrm{x}}) \epsilon_{a b} \epsilon^{i j} \partial_{j} \widetilde{\mathrm{x}}^{b} \partial_{i} \widetilde{\mathrm{x}}^{a} \Lambda
$$

Moreover, since this charge is conserved for any function $\Lambda$, its integrand is conserved, i.e., the magnitude

$$
\widetilde{B}(\widetilde{\mathrm{x}}) \epsilon_{a b} \epsilon^{i j} \partial_{j} \widetilde{\mathrm{x}}^{b} \partial_{i} \widetilde{\mathrm{x}}^{a}=2 \widetilde{B}(\widetilde{\mathrm{x}})\left|\partial_{i} \widetilde{\mathrm{x}}^{j}\right|=2 \widetilde{B}(\widetilde{\mathrm{x}})
$$

is conserved. Here in the last equality we used the constraint (4.14). We can fix its value according to the initial condition as

$$
\widetilde{B}(\widetilde{\mathrm{x}})=\left.\widetilde{B}(\widetilde{\mathrm{x}})\right|_{t=0}=\widetilde{B}(\widetilde{y}) .
$$

Note that this relation implies that the value of the magnetic field at the particle position is the same along all its motion. In other words, the particle moves along the level lines of the function $\widetilde{B}$. Again, this may be interpreted as a behavior analogous to that of the Chern-Simons theory, in which excitations move along the boundary, and can be described by a chiral boson. Since in our case the boundary has been replaced by a smooth space-dependent external field, this translates into a motion along the level lines of this external field, and the corresponding chiral boson theory will necessarily have to take this into account.

\section{SOLUTION OF THE CONSTRAINT AND THE RESULTING BOUNDARY THEORY}

\section{A. Solution of the constraint as a gradient expansion}

The next step is to solve the constraint (4.14) and rewrite the action in terms of the dynamical degrees of freedom. Here and in what follows, we use as parameters the filling fraction in the $\widetilde{\mathrm{x}}$ plane, defined as $\widetilde{\nu}=\tilde{\rho} / e \widetilde{B}$, and the effective (dimensionfull) parameter $\theta=1 /\left(2 \pi \widetilde{\rho}_{o}\right)$ defined in Ref. 18 as the square of the noncommutativity length.
Since our theory is defined only for wavelengths of the field $\widetilde{\mathrm{X}}^{k}$ that are large compared to the interparticle distance, it is natural to solve the constraint by a gradient expansion. The solution can be obtained by performing on the initial configuration $\widetilde{\mathrm{x}}^{k}=\tilde{y}^{k}$, an iteration of small area preserving diffeomorphisms of the form (4.12) (with $\Omega=0$ ). Calling $\theta \phi=\Lambda$ we get

$$
\widetilde{\mathrm{x}}^{k}=\widetilde{y}^{k}+\theta \epsilon^{k l} \partial_{l} \phi+\frac{\theta^{2}}{2} \epsilon^{k l} \epsilon^{i j} \partial_{i} \phi \partial_{j} \partial_{l} \phi+\mathcal{O}\left(\theta^{3}\right),
$$

then Eq. (4.14) is solved up to order $\theta^{3}$. This field represents the effective degrees of freedom after projection into the ground state. It can be interpreted to first order in $\theta$ as representing the coordinate fluctuations, i.e., the small displacement of the system along the flat directions of the potential. Incidentally we see in Eq. (5.1) that $\phi$ is defined up to the addition of an arbitrary function of time $\phi \rightarrow \phi+g(t)$, which is the usual gauge invariance of the chiral boson theory.

Replacing this solution in the action (4.16) we get

$$
\begin{aligned}
S= & \frac{1}{8 \pi^{2}} \int d t d^{2} \tilde{y} \widetilde{\nu}^{-1} \epsilon^{i j}\left(\partial_{j}\left(\phi \partial_{i} \partial_{t} \phi\right)+\frac{\theta}{3} \partial_{j}\left(\epsilon^{k l} \partial_{k} \partial_{t} \phi \partial_{i} \phi \partial_{l} \phi\right)\right) \\
& +\mathcal{O}\left(\theta^{2}\right) .
\end{aligned}
$$

\section{B. Boost back}

Note that the variables $\widetilde{x}^{i}(\tilde{y}, t)$ refer to the position of the particle initially located at $\tilde{y}$. This is the Lagrangian description of a fluid, where each fluid element is labeled with its initial position and then followed along its motion throughout the plane. However, the initial position $\tilde{y}^{i}$ is given in an awkward coordinate system that is related to that of the laboratory by the transformation discussed in Sec. III A. We would like to refer things to the laboratory coordinate system by going back on the original frame as $y^{i}=y^{i}(\widetilde{y})$. Note that for convenience we do not apply this operation to the $\widetilde{\mathrm{x}}^{i}$ fields. They still correspond to the position at time $t$ in the tilted system, but now the fluid element is identified by its initial position $y^{i}$ on the laboratory frame.

Under this transformation, the action becomes

$$
\begin{gathered}
\frac{1}{8 \pi^{2}} \int d t d^{2} y \nu^{-1} \epsilon^{i j}\left(\partial_{j}\left(\phi \partial_{i}\left(\partial_{t}-v^{a} \partial_{a}\right) \phi\right)\right. \\
\left.+\frac{\theta}{3} \partial_{j}\left(\epsilon^{k l} \partial_{k}\left(\partial_{t}-v^{a} \partial_{a}\right) \phi \partial_{i} \phi \partial_{l} \phi\right)\right)
\end{gathered}
$$

where we defined the position dependent filling fraction in the (physical) y plane as $\nu=\rho / e B$, and it satisfies

$$
\nu=\frac{\tilde{\rho}_{o}}{e B}\left(1+\frac{\left(\partial_{i} u\right)^{2}}{2 B^{2}}\right)
$$

On the other hand, the velocity of the excitations is defined as in Eq. (3.1)

In the special case in which $\nu$ is constant the action is a total derivative. The usual procedure is to assume this and then impose a boundary to the region in which the fluid moves. We choose a different way here, allowing the param- 
eter $\nu$ to change in space and introducing through it the information about the geometry, without adding any boundary. A further integration by parts gives

$$
\begin{aligned}
S= & -\frac{1}{8 \pi^{2}} \int d t d^{2} y t^{i}\left(\phi \partial_{i}\left(\partial_{t}-v^{a} \partial_{a}\right) \phi\right. \\
& \left.+\frac{\theta}{3} \epsilon^{b c} \partial_{b}\left(\partial_{t}-v^{a} \partial_{a}\right) \phi \partial_{i} \phi \partial_{c} \phi\right),
\end{aligned}
$$

where we defined the vector field $t_{i}(y)$, tangent to the level line of $\nu$ at $y$, as

$$
t^{i}=\epsilon^{i j} \partial_{j}\left(\nu^{-1}\right) .
$$

We see that the degrees of freedom are localized at the positions at which the change of variables discussed in Sec. III A is nontrivial, i.e., where the external electric field $\partial_{i} u$ is nonconstant. Moreover they propagate in the direction of $t_{i}$ along which $E^{2}$ is constant.

Here we see that, wherever the sample is homogeneous, the derivatives in front of each term vanish, leaving us without any dynamics. On the other hand, the dynamical degrees of freedom localize at places where there is a change in the properties of the material (i.e., the filling fraction). This is precisely what happens at the edge of the sample, and it is at the core of our argument.

To stress this point, let us suppose that the filling fraction changes as a step function, then their derivatives will provide delta functions leading us to a one-dimensional boundary theory, which corresponds to a Chiral Luttinger liquid description. ${ }^{9}$ Our treatment, however, is more general in the sense that it includes the possibility of dealing with smooth edges.

The action (5.5) is written completely in terms of the dynamical degrees of freedom $\phi$. Note that this is not a boundary theory because it is defined in the full twodimensional space. Nevertheless, the degrees of freedom are bounded to the region in which there is a change in the parameter $\nu$ and, as predicted, propagate chirally along their level surfaces.

\section{RECOVERING THE DISCRETE STRUCTURE NEAR THE EDGE}

When we approach the edge, the derivatives of the functions entering into the action are no longer negligible. Then the assumption we made when we took the continuum limit (4.4) is not satisfied, and the solutions of the continuum theory do not represent faithfully the underlying discrete system. To go around this problem, we need to recover the discrete structure near the edge. Calling $y_{\|}$the direction that runs along the edge and $y_{\perp}$ the perpendicular direction, we see that the $y_{\perp}$ derivatives become big in the edge region. Since $y_{\|}$derivatives are still small, we can keep the $y_{\|}$integral while in the direction $y_{\perp}$ transverse to the edge, we proceed as follows.

(1) Cut the space in the $y_{\perp}$ direction in $\mathcal{N}$ slices at $y_{\perp}^{n}$ of width $\Delta y_{\perp} \sim a$, where $a$ is the mean interparticle distance.
(2) Replace the integral by a sum over the values of the integrand evaluated at each slice, and define $\phi_{n}\left(y_{\|}\right)$ $\equiv \phi\left(y_{\|}, y_{\perp}^{n}\right)$.

(3) Replace all the $y_{\perp}$ derivatives by its finite difference approximation

$$
\partial_{\perp} \phi\left(y_{\|}, y_{\perp}^{n}\right)=\left[\phi\left(y_{\|}, y_{\perp}^{n}\right)-\phi\left(y_{\|}, y_{\perp}^{n-1}\right)\right] / \Delta y_{\perp} .
$$

With this method we obtain an effective theory for the fields $\phi_{n}\left(y_{\|}\right)$living on each slice $n$, with action

$$
\begin{aligned}
S= & -\frac{1}{8 \pi^{2}} \int d t d y_{\|} \sum_{n=0}^{\mathcal{N}}\left(\kappa_{n} \mathcal{L}_{\mathcal{F}}\left(\phi_{n}\right)+\kappa_{n}^{\prime}\left(\mathcal{L}_{\mathcal{S}}\left(\phi_{n}\right)\right.\right. \\
& \left.\left.+\mathcal{L}_{\mathcal{I}}\left(\phi_{n}, \phi_{n-1}\right)\right)\right),
\end{aligned}
$$

where we have defined the free, self-interaction, and interaction Lagrangians as

$$
\begin{gathered}
\mathcal{L}_{\mathcal{F}}\left(\phi_{n}\right)=\phi_{n} \partial_{\|}\left(\partial_{t}-v_{n} \partial_{\|}\right) \phi_{n}, \\
\mathcal{L}_{\mathcal{S}}\left(\phi_{n}\right)=\phi_{n}\left(\partial_{t}-v_{n} \partial_{\|}\right)\left(\partial_{\|} \phi_{n}\right)^{2}, \\
\mathcal{L}_{\mathcal{I}}\left(\phi_{n}, \phi_{n-1}\right)=-\phi_{n-1}\left(\partial_{t}-v_{n} \partial_{\|}\right)\left(\partial_{\|} \phi_{n}\right)^{2},
\end{gathered}
$$

and the constants $\kappa_{n}$ and $\kappa_{n}^{\prime}$ are given by

$$
\kappa_{n}=\frac{1}{\nu_{n}}-\frac{1}{\nu_{n-1}}, \quad \kappa_{n}^{\prime}=\frac{\theta}{2 \Delta y_{\perp}}\left(\frac{1}{\nu_{n}}-\frac{1}{\nu_{n-1}}\right) .
$$

It is important to note that the field $\phi_{n}$ will enter into the action only when the constants $\kappa_{n}$ and $\kappa_{n}^{\prime}$ are nonvanishing, i.e., if there is a change in the properties of the sample between the slices $n$ and $n+1$.

The gauge invariance is now $\phi_{n} \rightarrow \phi_{n}+g(t)$ adding the same $g(t)$ to all the $\phi$ 's.

Note that when the sample has a sharp edge, i.e., if the density changes within a region whose width $W$ is smaller than the slicing length $\Delta y_{\perp}$, i.e., $W<\Delta y_{\perp} \sim a$, then the whole procedure is not applicable and the boundary theory corresponds to the usual chiral boson theory. ${ }^{9}$

Let us suppose that the edge is wider than the slicing length, so that we have to keep a finite number of terms of the sum in Eq. (6.1), $n=1, \ldots, \mathcal{N}$.

We have then a single nondynamical field $\psi \equiv \phi_{-1}$ in the interaction term $\mathcal{L}_{I}\left(\phi_{0}, \phi_{-1}\right)$, all other fields appearing in the $\mathcal{L}_{I}\left(\phi_{n}, \phi_{n-1}\right)$ terms being dynamical. The integration of this multiplier field will enforce a constraint on the field $\phi_{0}$ to which it is coupled. When solved, $\phi_{0}=f\left(y_{\|}+v_{0} t\right)$, and replaced in the action, it implies that the interaction term with $\phi_{1}$ takes the form

$$
-\frac{1}{8 \pi^{2}} \kappa_{1}^{\prime} \int d t d y_{\|} f\left(y_{\|}+v_{0} t\right)\left(\partial_{t}-v_{1} \partial_{\|}\right)\left(\partial_{\|} \phi_{1}\right)^{2},
$$

which is different from zero provided that the two velocities are not equal. Since we have assumed that the electric field in the edge zone is approximately constant in regions of size $a \sim \Delta y_{\perp}$, hence $v_{0} \approx v_{1}$ and we can discard this term within the present approximation.

We have then obtained an action very similar to the original one but without any Lagrange multiplier 


$$
\begin{aligned}
S= & -\frac{1}{8 \pi^{2}} \int d t d y_{\|} \sum_{n=1}^{\mathcal{N}}\left(\kappa_{n} \mathcal{L}_{\mathcal{F}}\left(\phi_{n}\right)\right. \\
& \left.+\kappa_{n}^{\prime}\left(\mathcal{L}_{\mathcal{S}}\left(\phi_{n}\right)+\left(\delta_{n 1}-1\right) \mathcal{L}_{\mathcal{I}}\left(\phi_{n}, \phi_{n-1}\right)\right)\right),
\end{aligned}
$$

where the lower bound of the sum has changed and the coefficient $\left(\delta_{n 1}-1\right)$ ensures that there is no interaction term for the field $\phi_{1}$.

\section{APPLICATION OF THE MODEL: TUNNELING EXPONENTS}

Let us consider the case of an almost sharp boundary, in which the density can be well approximated by choosing $\mathcal{N}=1$. In that case the action is

$$
S=-\frac{1}{8 \pi^{2}} \int d t d y_{\|}\left(\kappa \phi \partial_{\|}\left(\partial_{t}-v \partial_{\|}\right) \phi+\kappa^{\prime} \phi\left(\partial_{t}-v \partial_{\|}\right)\left(\partial_{\|} \phi\right)^{2}\right),
$$

where we have dropped the subindices since we have a single field. This is a chiral boson theory with a cubic higher derivative self-interaction, the latter arising essentially from the smoothness of the edge. To see the effect of this last term in the physical properties, we compute the propagator since it is directly related to the tunneling exponent. ${ }^{9}$

In Fourier space we write for the Feynman propagator

$$
G\left(p, \omega_{p}\right)=\frac{2 \pi}{\kappa p\left(\omega_{p}-v p\right)+i \epsilon},
$$

while the vertex is proportional to

$$
-\kappa^{\prime} \delta(p+q+r)\left(\omega_{p}-v p\right) q r .
$$

Then, the one loop corrected propagator reads

$$
G^{\prime}\left(p, \omega_{p}\right)=\frac{2 \pi}{\kappa p\left(\omega_{p}-v p\right)-\delta G\left(p, \omega_{p}\right)} .
$$

We consider those Feynmann diagrams whose contribution to the corrected propagator is

$$
\delta G\left(p, \omega_{p}\right)=\pi \Lambda^{2} \frac{\kappa^{\prime 2}}{\kappa^{2}}\left(\omega_{p}-v p\right) p,
$$

where $\Lambda$ is a momentum cutoff. Replaced in Eq. (7.2) this gives

$$
G\left(p, \omega_{p}\right)=\frac{1}{(\kappa+\delta \kappa) p\left(\omega_{p}-v p\right)},
$$

where the nonuniversal correction to the level is given by

$$
\delta \kappa=-\pi \frac{\kappa^{\prime 2}}{\kappa^{2}} \Lambda^{2}
$$

Note that the effective coupling is $\kappa_{n}^{\prime 2} / \kappa_{n}^{2}=\theta^{2} / 4 \Delta x_{\perp}^{2}$, being independent of the details of the boundary. A nonvanishing correction to the velocity arises from the remaining diagrams but it produces no physical consequences.

Since the tunneling exponent is directly related to the level by $\alpha \approx \kappa$, the appearance of a correction for the latter implies, to first order, a correction to the tunneling exponent. It should be stressed at this point that the correction is negative, in qualitative accordance with the experimental results. This is the main result of this section.

To estimate the magnitude of this correction, we need to relate the momentum cutoff $\Lambda$ to the minimal space distance measurable $a$, which naturally leads to the identification $\Lambda$ $=\pi / a$. Using Eq. (6.3) we then get for this choice of the cutoff

$$
\delta \kappa=-\frac{\pi}{\left(4 a^{2} \tilde{\rho}_{o}\right)^{2}}=-\frac{\pi}{16} \approx-0.196,
$$

where we have further identified $\tilde{\rho}_{o}=1 / a^{2}$. The predicted dependence on the density of this nonuniversal correction could in principle be tested experimentally.

Putting it all together leads to a linear dependence of the tunneling exponent $\alpha$ as a function of $1 / \nu$ which is very close to the experimental fit presented in Ref. 10 (see, for example, Fig. 3 of this reference). However, it should be kept in mind that our present result is valid for incompressible situations. It can be observed that the departure of our result from the experimental data becomes greater for increasing $1 / \nu$, which could be attributed to the need to work with $\mathcal{N}$ $>1$ in Eq. (6.5). This could in turn be related to the need to consider a wider boundary region. The analysis of the higher $\mathcal{N}$ case will be presented separately. ${ }^{19}$

\section{CONCLUSIONS AND OUTLOOK}

To summarize, we have constructed an effective field theory that describes the boundary region of a fractional quantum Hall effect droplet, starting from the microscopic description. We included the interplay between the granularity of the fluid (the interparticle distance $a$ ) and the effects of a smooth boundary. This extended boundary has been defined by means of a nonvanishing electric field that leads to a space-dependent filling fraction within this region. The physical excitations of the system are localized at the boundary, and are described by a system of self-interacting chiral bosons.

All the approximations we made along our calculations are under control: by taking the nonrelativistic limit in the strong magnetic field regime, we enforced the projection into the LLL. Keeping the interaction between particles completely generic, this low energy projection results in a constraint on the dynamical degrees of freedom of the model. With the help of the symmetries of the system, we were able to replace this constraint by an equivalent one that implies incompressibility and is completely independent of the details of the interaction potential.

As an example of the possible applications of our approach, we have computed the correction to one loop order of the tunneling exponent to lowest order in the boundary width, which is in qualitative agreement with experimental results.

The results presented here can in principle be improved in a systematic way. Wider boundary regions can be easily treated within the present approach, by simply taking higher values of $\mathcal{N}$ in Eq. (6.5). In this case, we get a new self- 
interacting chiral boson on each slice, which is coupled to the immediately inner slice. For example, for two slices we have

$$
\begin{aligned}
S= & -\frac{1}{8 \pi^{2}} \int d t d y_{\|}\left(\kappa_{1} \mathcal{L}_{\mathcal{F}}\left(\phi_{1}\right)+\kappa_{2} \mathcal{L}_{\mathcal{F}}\left(\phi_{2}\right)+\kappa_{1}^{\prime} \mathcal{L}_{\mathcal{S}}\left(\phi_{1}\right)\right. \\
& \left.+\kappa_{2}^{\prime}\left(\mathcal{L}_{\mathcal{S}}\left(\phi_{2}\right)-\mathcal{L}_{\mathcal{I}}\left(\phi_{2}, \phi_{1}\right)\right)\right) .
\end{aligned}
$$

If we want to evaluate as before the correction to the level of the chiral boson that lives on the outer slice, we would now have additional diagrams coming from the interaction with the inner boson. It is easy to see that the resulting diagrams involve only corrections to the velocity, and do not affect the level. In particular, this implies that tunneling exponents remain unchanged.

Higher loop computations could also be envisaged; however, they would involve the solution of the constraint to higher orders in $\theta$. Indeed, a two loop computation includes powers of $\theta^{4}$, which were discarded when solving the constraint by formula (5.1). A consistent calculation then needs the inclusion of these terms in Eq. (5.1), which will in turn give rise to additional terms in the Lagrangian. This is out of the scope of the present paper.

As a further improvement, one can describe compressible situations by including the effects of the degrees of freedom transverse to the flat directions of the potential [described by $\Omega$ in Eq. (4.13)]. To this end, instead of writing the incompressibility constraint (4.14) and solving it by Eq. (5.1), we can simply parametrize a small deviation of the system from its ground state as

$$
\widetilde{x}^{i}(\tilde{y}, t)=\tilde{y}_{j}+\theta\left(\epsilon_{j k} \partial_{k} \phi+\partial_{j} \omega\right)+O\left(\theta^{2}\right),
$$

where $\theta \omega=\Omega$. This parametrization is completely general, since it includes deviations $\omega$ that are transverse to the flat directions of the potential. Higher orders in $\theta$ can be included as in Eq. (5.1), even if for simplicity we will not write them in what follows. The resulting form of the action is

$$
\begin{aligned}
& \frac{1}{8 \pi^{2}} \int d t d^{2} y\left(t^{i}\left(\phi \partial_{i}\left(\partial_{t}-v^{a} \partial_{a}\right) \phi+\omega \partial_{i}\left(\partial_{t}-v^{a} \partial_{a}\right) \omega\right)\right. \\
& \quad+2 \nu^{-1} \partial_{i} \phi \partial_{i}\left(\partial_{t}-v^{a} \partial_{a}\right) \omega \\
& \left.\quad-\int d^{2} y^{\prime} f^{i j}\left(y, y^{\prime}\right) \partial_{i} \omega(y) \partial_{j} \omega\left(y^{\prime}\right)\right) .
\end{aligned}
$$

We see that the first two terms are proportional to $t^{i}$ and hence localized at the boundary, while the third integrates over the whole sample. The last nonlocal term comes from a functional expansion of the interaction potential $V[\tilde{x}]$ in powers of $\omega$, where $f^{i j}\left(y, y^{\prime}\right)=\delta^{2} V / \delta \mathrm{x}_{i}(y) \delta \mathrm{x}_{j}\left(y^{\prime}\right)$. Even if it is evident that this is a very complicated system, we will try to convince the reader that it deserves further investigation. We will specialize it to a straight boundary as before, and explore the simple case of a static configuration with vanishing $\phi$. Taking advantage of the isotropy of the interparticle interactions we write $f^{i j}\left(y, y^{\prime}\right)=\delta^{i j} f\left(y, y^{\prime}\right)$. Moreover, we can expand this nonlocal potential in powers of its typical nonlocality range $\ell$ as $f\left(y, y^{\prime}\right)=f_{o}\left[\delta\left(y-y^{\prime}\right)+\ell^{2} \partial^{2} \delta\left(y-y^{\prime}\right)+\cdots\right] .^{21}$ The equations of motion in this limit read

$$
\begin{gathered}
t \partial_{\perp}\left(v \partial_{\|} \omega\right)-\nu^{-1} \partial^{2}\left(v \partial_{\|} \omega\right)=0, \\
-t v \partial_{\|}^{2} \omega+f_{o}\left(\partial^{2} \omega+\ell^{2} \partial^{2} \partial^{2} \omega\right)=0,
\end{gathered}
$$

here $t$ and $v$ are the moduli $t^{i}$, and $v^{a}$, respectively. The first equation is solved by $\omega=\omega\left(y_{\perp}\right)$. The second equation immediately imply $\partial^{2} \omega \propto \sin \left(\ell y_{\perp}+\delta\right)$. This result becomes suggestive when we note that under the small deviation of the incompressible ground state parametrized by $\omega$, the density scales as $\rho=\widetilde{\rho}_{o}\left(1+\partial^{2} \omega\right)$. Then as we move in the direction transverse to the boundary, we see an oscillating behavior of the density that may in principle be related to the so-called boundary reconstruction effect. These are very preliminary results, and should be intended not as a proof but just as an insight of the possibilities of the present approach.

Other approaches to include a boundary in a ChernSimons description of a quantum Hall droplet have been proposed. ${ }^{20}$ In this context, it would be interesting to study their connection to our model.

It should be mentioned that the experimental data in Ref. 10 were reanalyzed in Ref. 13 in view of the results of Ref. 15 , and certain degree of agreement with the plateau structure was obtained. Here we propose an alternative description which provides a reasonable agreement with the raw experimental data as presented in Ref. 10. It would be interesting to study the higher order theory that we have constructed in the present paper along the lines of the approach presented in Ref. 15. This could provide a closer agreement between theoretical and experimental results.

It would be also interesting to analyze the consequences of the higher order corrections induced by the smoothness of the boundary that we have obtained here in the transition between plateau states.

\section{ACKNOWLEDGMENTS}

We thank A. Dobry, E. Fradkin, J. Polonyi, P. Pujol, G. L. Rossini, and G. Silva for helpful discussions. N.E.G. is grateful to SISSA and ICTP for hospitality and financial support during the early stages of this work. This work was partially supported by ECOS-Sud Argentina-France collaboration (Grant No. A04E03) and PICS CNRS-CONICET (Grant No. 18294), and ISF grant INSTANS. 
*cabra@1pt1.u-strasbg.fr

†grandi@fisica.unlp.edu.ar

${ }^{1}$ K. v. Klitzing, G. Dorda, and M. Pepper, Phys. Rev. Lett. 45, 494 (1980).

${ }^{2}$ D. C. Tsui, H. L. Stormer, and A. C. Gossard, Phys. Rev. Lett. 48, 1559 (1982).

${ }^{3}$ R. B. Laughlin, Phys. Rev. Lett. 50, 1395 (1983).

${ }^{4}$ R. de-Picciotto, M. Reznikov, M. Heiblum, V. Umansky, G. Bunin, and D. Mahalu, Nature (London) 389, 162 (1997).

${ }^{5}$ J. K. Jain, Phys. Rev. Lett. 63, 199 (1989).

${ }^{6}$ S. C. Zhang, T. H. Hansson, and S. Kivelson, Phys. Rev. Lett. 62, 82 (1989); S. Girvin, R. E. Prange, and S. M. Girvin, The Quantum Hall Effect (Springer-Verlag, Berlin, 1990); E. Fradkin Field Theories of Condensed Matter Systems (Addison-Wesley, Reading, MA, 1991).

${ }^{7}$ A. M. Chang, Rev. Mod. Phys. 75, 1449 (2003).

${ }^{8}$ D. C. Cabra and N. E. Grandi, arXiv:cond-mat/0511674 (unpublished).

${ }^{9}$ X.-G. Wen, Adv. Phys. 44, 405 (1995).

${ }^{10}$ M. Grayson D. C. Tsui, L. N. Pfeiffer, K. W. West, and A. M. Chang, Phys. Rev. Lett. 80, 1062 (1998).

${ }^{11}$ K. Yang, Phys. Rev. Lett. 91, 036802 (2003).
${ }^{12}$ X. Wan, F. Evers, and E. H. Rezayi, Phys. Rev. Lett. 94, 166804 (2005).

${ }^{13}$ A. M. Chang, M. K. Wu, C. C. Chi, L. N. Pfeiffer, and K. W. West, Phys. Rev. Lett. 86, 143 (2001).

${ }^{14}$ A. Würtz, R. Wildfeuer, A. Lorke, E. V. Deviatov, and V. T. Dolgopolov, Phys. Rev. B 65, 075303 (2002); Y. N. Joglekar, H. K. Nguyen, and G. Murthy, ibid. 68, 035332 (2003); D. B. Chklovskii, B. I. Shklovskii, and L. I. Glazman, ibid. 46, 4026 (1992); D. B. Chklovskii, ibid. 51, 9895 (1995).

${ }^{15}$ C. de C. Chamon and E. Fradkin, Phys. Rev. B 56, 2012 (1997).

${ }^{16}$ C. de C. Chamon and X.-G. Wen, Phys. Rev. B 49, 8227 (1994); D.-H. Lee and X.-G. Wen, arXiv:cond-mat/9809160 (unpublished).

${ }^{17}$ William H. Press, Saul A. Teukolsky, William T. Vetterling, and Brian P. Flannery, Numerical Recipes (Cambridge University Press, Cambridge, 2007).

${ }^{18}$ L. Susskind, arXiv:hep-th/0101029 (unpublished).

${ }^{19}$ D. C. Cabra and N. Grandi (unpublished).

${ }^{20}$ A. P. Polychronakos, J. High Energy Phys. 0104, 011 (2001).

${ }^{21}$ That this is the correct expansion can be checked by Fourier transforming the double integral and expanding in powers of the wave number. 